\title{
Agricultural level of development: a comparative study between project and non-project area of JSW Bengal Steel Ltd. at Sundra Basin of Salboni block, Paschim Medinipur, West Bengal, India
}

\author{
Sumanta Prakash Shee* and Ramkrishna Maiti*
}

\begin{abstract}
M/S JSW Bengal Steel Ltd. is one of the India's leading steel producer company is going to set up a 10.0 Mtpa (Million ton per annum) and 300 MW Captive Power Plant by 2020 at Sundra Basin of Paschim Medinipur, but the proposed projet has been suspended due to lack of raw materials on 1st December 2014. For the said project $17.1 \mathrm{sq} \mathrm{km} \mathrm{land}$ has been acquired from 22 mouzas in 2009. After land acquisition an extensive deterioration in the agricultural level of development has been observed at the JSW project affected mouzas. Due to losing of productive land and profession of primary activities, people of project sites have been suffering from steep reduction in income and employment. As a consequence, a sharp difference in development between project affected and non-affected areas has been observed at basin scale. The value of composite index of agricultural development varies from 0.28 in 2007 to 0.94 in 2014 that indicates a wide regional disparities present at study area and requires immediate attention for all inclusive development. So there is an immediate need for proper planning on project affected mouzas to make alternative livelihood opportunities with help of local NGOs and Government.
\end{abstract}

Keywords: Composite index, JSW Bengal Steel Ltd, Agricultural development, Land acquisition

\section{Introduction}

Quality of life of the people should be improved through proper development planning (Inglehart 1997; Myrdal 1972; Ohlan 2013). Development project may alter existing physical and social situation of the concerned region and its surroundings that may bring unanticipated adverse impacts (Long et al. 2007; Madon 2000; Szirmai 2015) on society. In order to manage the adverse impact, a development indicator is needed to develop for bringing about uniformity in regional development (Akama and Kieti 2007; Choudhury 1992; Dasgupta 1971; Sarker 1994). Development is a multi-dimensional process (Bhattacharya and Mahalanobis 1967; Dreze and

\footnotetext{
*Correspondence: sumantaprakashshee@gmail.com; ramkrishnamaiti@ yahoo.co.in

Vidyasagar University, Paschim Medinipur, West Bengal 721102, India
}

Sen 1999) which cannot be fully estimated by a single indicator. Moreover, when we use a number of indicators individually, we cannot get any integrated and easily comprehensive picture of reality. So, it necessitates to build up a composite index (CI) of development based upon optimal combination of different developmental indicators in the analysis for assessing the level of development (Demurger 2001; Florek et al. 1951; Narain et al. 2011). Drewnowski (1972) has defined an easily accessible socio-economic indicator which contains information about the degree of satisfaction of human needs.

Several studies have been done to provide various dimensions to concept of development (Choudhury 1995; Das 1999; Parihar and Srivastava 2003) and the studies revealed that there are wide disparities in the level of development among different regions. A deeper 
level analysis on the estimation of level of development has been done in different years on same ground for several states, e.g. Orissa (Narain et al. 1992, 1993), Andhra Pradesh (Narain et al. 1994a, b, 2009), Kerala (Narain et al. 1994a, b, 2005), Utter Pradesh (Narain et al. 1995), Maharashtra (Narain et al. 1996), Karnataka (Narain et al. 1997, 2003), Tamil Nadu (Narain et al. 2000), Madhya Pradesh (Narain et al. 2002), Assam (Narain et al. 2004), West Bengal (Narain et al. 2011; Rai and Bhatia 2004, 2011) and Jammu and Kashmir (Narain et al. 1991). It is observed that entire parts of low developed districts are not backward but there are some parts which are also better development (Raja and Yousaf 2014). In addition on the base of above studies, researchers have been trying to provide various dimensions to the concept of development.

M/S JSW Bengal Steel Ltd. of Jindal group, part of O.P. Jindal is one of the India's leading steel producers with a significant presence in sector like mining, stainless steel, carbon steel, large diameter pipes, non-ferrous metals, power generation and distribution and maritime infrastructure (Jindal Steel and Power Ltd 2014; Sudalaimuthu and RAJ 2009). JSW Steel Ltd. operates a steel plant at Vijayanagar district, Bellary in Karnataka state. The JSW group also owns and operates southern Iron \& Steel Company Ltd. which is only integrated steel plant in Tamil Nadu and also cold rolling, galvanizing and color coating plants at Tarapur and Vasind in Maharashtra.

Now the company is signed a pact with West Bengal Government on 11th January 2007 to set up a $10.0 \mathrm{Mtpa}$ steel plant and 300 MW CPP by 2020 at Sundra basin of Paschim Medinipur district, West Bengal (JSW EIA 2007). The basin is stretching between $22^{\circ} 37^{\prime} 09^{\prime \prime} \mathrm{N}$ to $22^{\circ} 36^{\prime} 02^{\prime \prime} \mathrm{N}$ and $87^{\circ} 13^{\prime} 44^{\prime \prime} \mathrm{E}$ to $87^{\circ} 16^{\prime} 09^{\prime \prime} \mathrm{E}$ (Fig. 1 ) is one of the backward regions of this state. Sundra is the tributary of Tamal that ultimately pours to the river Silaboti. As of 2011 census, 32,689 persons have distributed among 109 smallest revenue units (mouzas) with a density of $274 / \mathrm{sq} \mathrm{km}$. The maximum concentration of population has been observed at Saiyadpur (1114 person), Godapiasol (1387 person), Jhar Bhanga (1983 person), Kontai (2102 person), Benachapra (1203 person), Dudiabandi (887 person), Bagbasa (903 person), Jamdedya (827 person) mouza, etc. along the road side due to accessibility of high transport facility. The literacy rate is $58 \%$. Scheduled Castes and Tribes constitute 24.1 and $16.3 \%$ of the

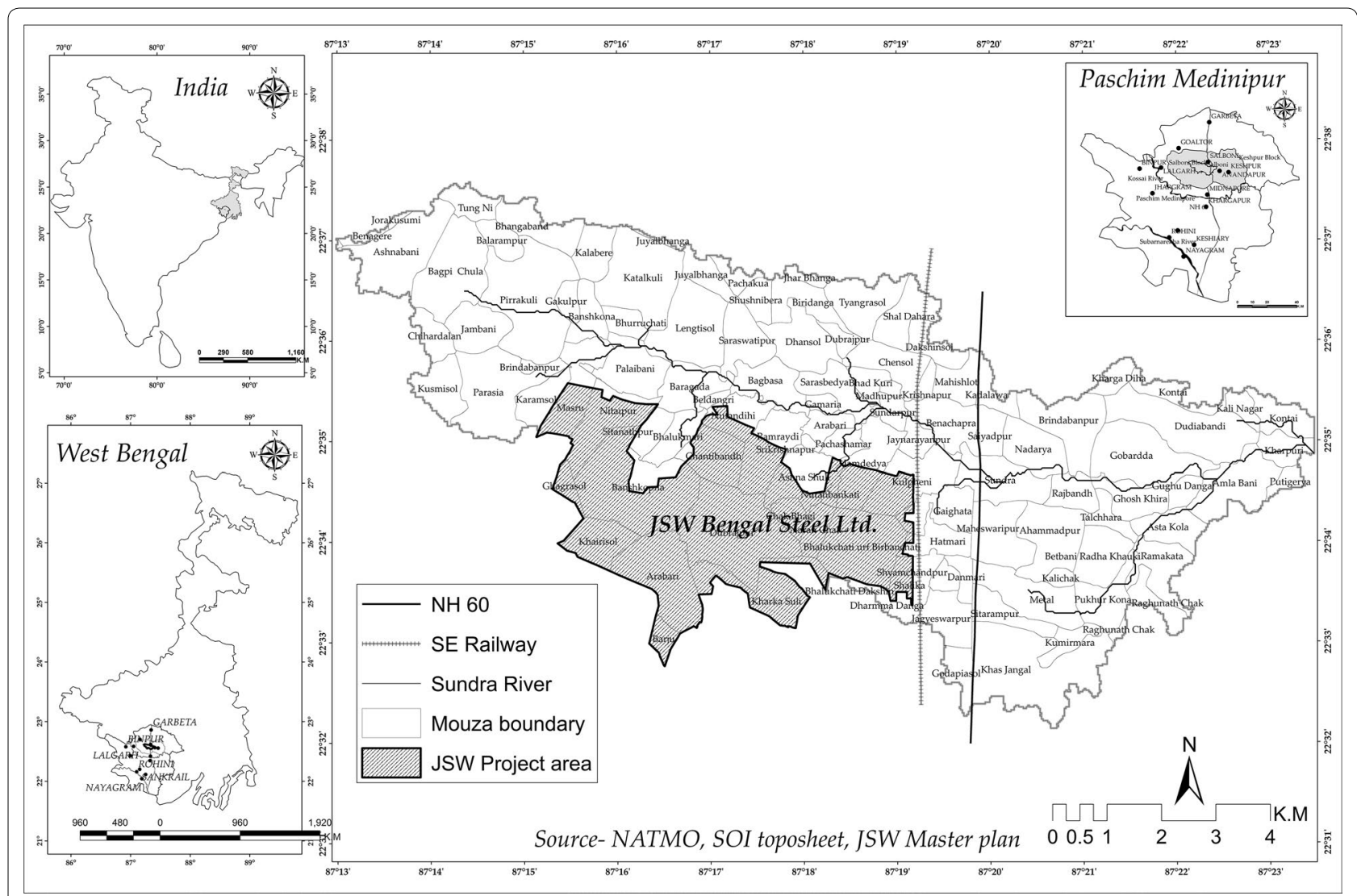

Fig. 1 Location of the study area 
population respectively (Census of India 2001, 2011). Agriculture, livestock production and collecting are the main stay of the local economies (District statistical handbook 2006, 2007).

For the proposed project $17.1 \mathrm{sq} \mathrm{km}$ land has been acquired from 22 mouzas in 2009. As a consequence a huge landuse conversion has been observed. The study area has lost $1.3 \mathrm{sq} \mathrm{km}$ of crop land, $9.6 \mathrm{sq} \mathrm{km}$ of forest land, $2.9 \mathrm{sq} \mathrm{km}$ of waste land and $3.3 \mathrm{sq} \mathrm{km}$ of fodder farm land. Livelihood of local residents of project site is mainly rely on natural resources like land and forest. But on 1st December 2014, JSW authority has announced to suspend the proposed project (Anandabazar, 2014) due to lack of raw materials. As a consequence the project affected household $(\mathrm{HH})$ are in severe condition in terms of their livelihood, income and accessibility. At Nutandihi, Ashna Shuli, Kharka Suli, Dubrajpur, Barju, Ghagrasol mouza there were 36, 124, 100, 14, 17 and 46 persons of main cultivators before land acquisition but after acquisition this figure is belong to $8,41,5,0,5$ and 7 persons respectively. Maximum percentage of marginal workers has been recorded at Nutandihi (54\%), Khairisol (58\%), Barju (53\%) while it was 0, 19 and $26 \%$ respectively before acquisition. At lower catchment Rajbandh, Bagpi Chula, Dudiabandi, Ghosh Khira, Amla Bani, Asta Kola, Putigerya, Kharpuri mouzas are depending on agricultural activities; but at project site Nutandihi, Nitaipur, Ashna Shuli, Kulpheni, Gaighata, Hatmari, Shalika, Kharka Suli, Arabari, Khairisol major source of income were agriculture (40-60\%) and livestock farming $(20-30 \%)$, now after land acquisition and due to clearance of forest cover this percentage has been observed as $20-25 \%$ and only $8-10 \%$ respectively.

The main objective of the study is to estimate a CI of development in agricultural sector for comparison in between pre (2007) and post (2014) land acquisition session. The outcome of level of development at mouza level will help to identify where a given mouza stands in relation to others.

\section{Data and method}

"Development is a multidimensional process and it cannot be fully estimated by single indicators. Moreover, a number of indicators when analyzed individually do not provide an integrated and easily comprehensive picture of reality" Narain et al. (2011). The major limitation arises from the assumption made about the developmental indicators themselves and their weightage in the aggregate index. To overcome this problem, a CI of development was constructed by Narain et al. (2011) to obtain a statistical method of determining homogenous units in an n-dimensional vectorial space. Hellwig (1967) of the Wroclow School of Economics applied taxonomy method for ranking and comparing between different countries. The method is very sensitive and valid measure of development levels, because it takes account of the dispersion among component indicators. According to Harbison et al. (1968) "it provides a useful tool for interpolation of statistical data sets up a measure of social and economic maturity and introduces a concept of pattern of development which may prove to be very useful in planning". Ewusi (1976) has applied the method to find out the disparities in levels of regional development in Ghana. Arief (1982) and Narain et al. (2011) have applied the similar methods in the studies conducted in West Bengal of India respectively. Bhatia and Rai (2004) have applied the method in small area to prepare a project report of planning commission of India. Indicators common to all mouzas of the study area have been included in the analysis for evaluating the level of development. We have gathered information of per capita irrigated and non-irrigated crop land, per capita crop land acquired for proposed project, crop income (in Rs) before and after land acquisition, yield rate of crops $(\mathrm{kg} /$ sq meter), consumption of fertilizer in $\mathrm{kg} / \mathrm{sq}$ meter, production of egg, milk etc. through HH survey (2007-2010 and 2012-2014) and data (mouza wise net sown area, total population, marginal workers, no of main cultivators etc.) of collected from secondary source such as Census of India $(2001,2011)$ and District statistical handbook $(2006,2007)$ have been used as input in this method. CI of development for project affected and nonproject affected mouzas have been obtained by using the following indicators.

\section{Agricultural development indicators}

- Net Sown Area (in sq m).

- No of Marginal Workers.

- Irrigated Area (in sq m).

- Crop Income (in Rs).

- No of Main Cultivators.

- Area not available for cultivation (in sq m).

- Area under cereal crops (in sq m).

- Area under rabi crops (in sq m).

- Cropping intensity (\%).

- Yield rate of total crops (kg/sq m).

- Production of total cereal (000 kg).

- Consumption of fertilizer in $\mathrm{kg} / \mathrm{sq} \mathrm{m}$.

- Production of egg (Hen + Duck) in hundreds.

- Production of milk (cow + goat + buffalo) in $\mathrm{kg}$.

A total of 14 indicators have been applied in this analysis, those are the major interacting indicators for agricultural development. A brief introduction of level of development method (Narain et al. 2011) used in this study has been discussed below. 


\section{Measuring the level of development}

Let $\left[X_{i j}\right]$ be the data matrix showing the variables of $i$ th mouza $(i=1,2, \ldots . n)$ and $j$ th indicator $(j=1,2, \ldots k)$.

Every mouza is represented by vector in a $k$-dimensional space. Since the units of measurement of the variables considered are not uniform, combined analysis $\left[X_{i j}\right]$ is transformed to the matrix of standardised indicators $\left[Z_{i j}\right]$ using following equation (Eq. 1.1)

$$
\left[Z_{i j}\right]=\frac{X_{i j}-\overline{X_{j}}}{\sigma_{j}}
$$

where, $\overline{X_{j}}=\frac{\sum_{i=1}^{N} X_{i j}}{N}$ and $\sigma_{j}=\left(\sum_{i=1}^{N}\left(X_{i j}-\overline{X_{j}}\right)^{2}\right)^{1 / 2}$

From $\left[Z_{i j}\right]$ the optimal value of each indicator was identified. Let it be denoted by $Z_{0 j}$. The optimal value will be either the maximum value or minimum value of the indicator depending upon the direction of the impact of an indicator on the level of development. For example, increase in literacy rate would positively affect the development, while increasing population density may adversely affected the development. For obtaining the pattern of development $C_{i}$ of the $i$ th mouza, square of the deviation of the individual value of a variate from the best value was calculated. In other words $P_{i j}$ was calculated using following Eq. 1.2

$$
P_{i j}=\left(Z_{i j}-Z_{o j}\right)^{2}
$$

For each $i$ and $j$ pattern of development is given by

$$
C_{i}=\left[\sum_{j=1}^{k} P_{i} /\left(c v_{j}\right)\right]^{1 / 2}
$$

where $\left(c v_{j}\right)=$ coefficient of variation of the $j$ th indicator in $X_{i j}$. CI 'measure of development' $\left(D_{i}\right)$ is given by

$$
D_{i}=C_{i} / C
$$

where

$$
C=\bar{C}+3 \sigma C_{i}
$$

where $\bar{C}=\frac{\sum_{i=1}^{N} C_{i}}{N}$ and $\sigma C_{i}=\left(\sum_{i=1}^{N}\left(C_{i}-\bar{C}\right)\right)^{1 / 2}$

The smaller $D_{i}$ will indicate more development and higher value of $D_{i}$ will indicate low level of development. The value of $D_{i}$ ranges between 0 and 1 .

\section{The level of development}

The composite indices of development for different mouzas of Sundra catchment has been estimated on the basis of development in agriculture, infrastructural and overall socio-economic sectors. The mouzas have been ranked on the basis of development indices. The composite indices of development along with the rank of mouzas have been discussed below.

For relative comparison among different mouzas within the study area regarding the level of development, a simple ranking of mouzas on basis of CI would be sufficient for classificatory purpose. Mouzas are classified in four stages of development as High level, High middle level, Low middle level and Low level. If the mouzas have CI less than or equal to (Mean - SD) (SD means Standard Deviation) are described as High level development and the mouzas having the $\mathrm{CI}$ greater than or equal to (Mean $+\mathrm{SD})$ are described as Low level development. If the CI lies in between (Mean) and (Mean - SD) are described as High middle level development and the mouzas having CI in between (Mean) and (Mean + SD) are described as Low middle level development (Ohlan 2013).

\section{Result and discussion}

Keeping in a view on detailed analysis of agricultural development in the study area between prior (2007) and post (2014) land acquisition, with help of following indicators a composite agricultural development index has been made along with their mouza wise ordinal rank. The study area mainly consists of 109 mouzas and within it 22 mouzas has been treated as project affected.

\section{Agricultural development indicators}

Percentage of net sown area Relative comparisons among the different mouzas of study area are made between 2007 and 2014 regarding net sown area. Out of 109 mouzas, 18 mouzas are found to have positive changes (15.2\%) and project affected 22 mouzas recorded negative changes $(-21.1 \%)$. Net sown area has been increased by $36.6 \%$ at Palaibani mouza, followed by Mahishlot (35.8\%), Saiyadpur (26.1\%), Dakshinsol (21.9\%), Gamaria (20.1\%), Brindabanpur (18.6\%), Nadarya (18.5\%), Ahammadpur (12.9\%), Kalichak (10.1\%), Asta Kola (9.9\%) mouza. The positive changes have been observed in only non-project affected mouzas (Fig. 2).

Project affected mouzas have lost their net sown area due to land acquisition in 2007. Maximum reduction in net sown area has been observed at Dubrajpur (73.6\%) followed by Chantibandh (87.1\%), Chak Bhagi (56.7\%), Ramraydi (51.4\%), Ashna Shuli (39.5\%) and Nitaipur (38.1\%) mouza.

Marginal workers Based on the census data it has been observed that marginal workers have increased rapidly by $17 \%$ within the study area. It was nearly $23.0 \%$ in 2001 and voluminous to $40.4 \%$ in 2011 . Figure 3 depicts the changing pattern of marginal workers within the study area. At lower catchment Rajbandh, Ahammadpur, Bagpi 


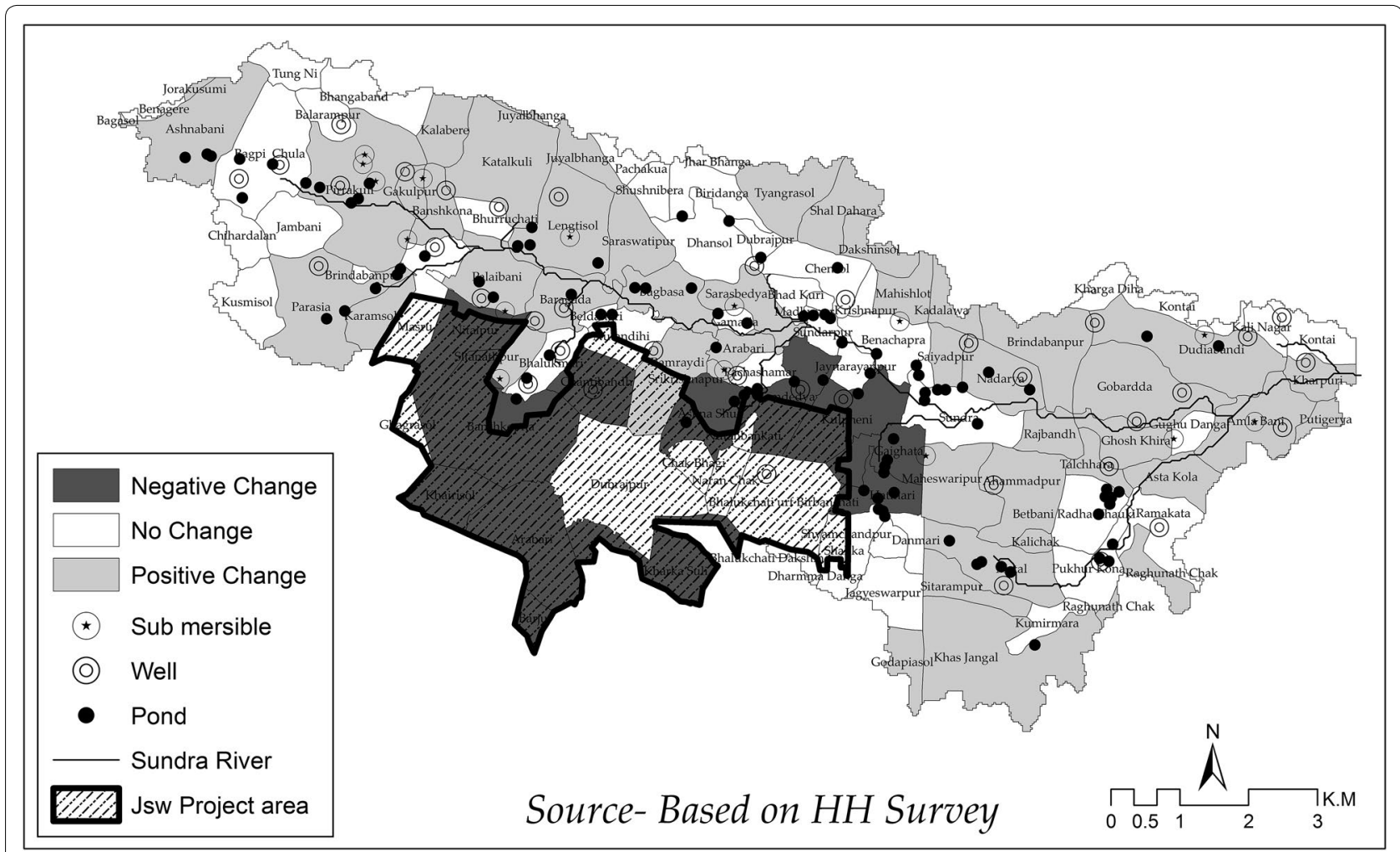

Fig. 2 Positive and negative changes in net sown area in between 2007 and 2014

Chula, Dudiabandi, Ghosh Khira, Gobardda, Amla Bani and Asta Kola mouza marginal workers have decreased by more than $20 \%$.

The marginal workers have increased by on an average of 26.5 and $15.0 \%$ respectively at project and non-project site. During that period total population are increased by $12.7 \%$ but average net sown area remains same. At upper and middle catchment due to lack of irrigation facility, poor soil quality, inadequate non-farm activity are contributing to increase number of marginal workers quite rapidly. From observation at Dakshinsol, Katalkuli, Krishnapur, Kalichak, Godapiasol, Jorakusumi, Benagere, Chensol, Jhar Bhanga, Pachakua and Kharga Diha mouza marginal workers became almost double from that of last census year. Among the project affected mouzas Khairisol records maximum share $(90 \%)$ of marginal workers, while before acquisition (2001) the figure was only $15.5 \%$. Other affected mouzas like Nutandihi (84.4\%), Arabari (75.3\%), Banshkopna (67.7\%), Chantibandh (58.1\%) and Ashna Shuli (61.2\%) also registered a huge percentage of marginal workers. Marginal workers are inversely related to agricultural development.

Percentage of irrigated area The net irrigated area with respect to total crop land within the study area has increased from $16.3 \%$ in 2007 to $18.5 \%$ in 2014. Mouza wise distribution of irrigated land has been projected in Fig. 4. It is observed that 32 mouzas experienced improve irrigation facility through bore well. Putigerya mouza is one of them, experiencing increase in net irrigated area by $40 \%$ in 2014. Ahammadpur (38.7\%), Sitanathpur (29.0\%), Saraswatipur (28.2\%), Gobardda (24.4\%), Pirrakuli (20.1\%), Dakshinsol (14.8\%), Asta Kola (12.7\%), Maheswaripur (11.4\%), Sarasbedya (10.5\%) mouza experience increase in net irrigated land area. But at the project affected mouzas (Fig. 5) net irrigated land area had been declined after acquisition of irrigated land for proposed project. The statistic reveals that the maximum irrigated land had been acquired from Banshkopna (16.6\%) followed by Ashna Shuli (16.4\%), Chantibandh (11.9\%), Hatmari (9.8\%) and Kulpheni $(9.1 \%)$ mouza. After acquisition of water bodies, as a consequence net irrigated area has been declined in the mouzas outside the project area.

Percentage of crop income Through detailed HH survey it is observed that crop income as a present to total income varies from upper catchment to lower catchment within the study area. Due to good quality of land and better irrigation facility at lower catchment crop income ranges from 57.0 to $80.0 \%$ while in upper catchment it varies from 24.0 to $40.0 \%$. Figure 6 depicts the variation 


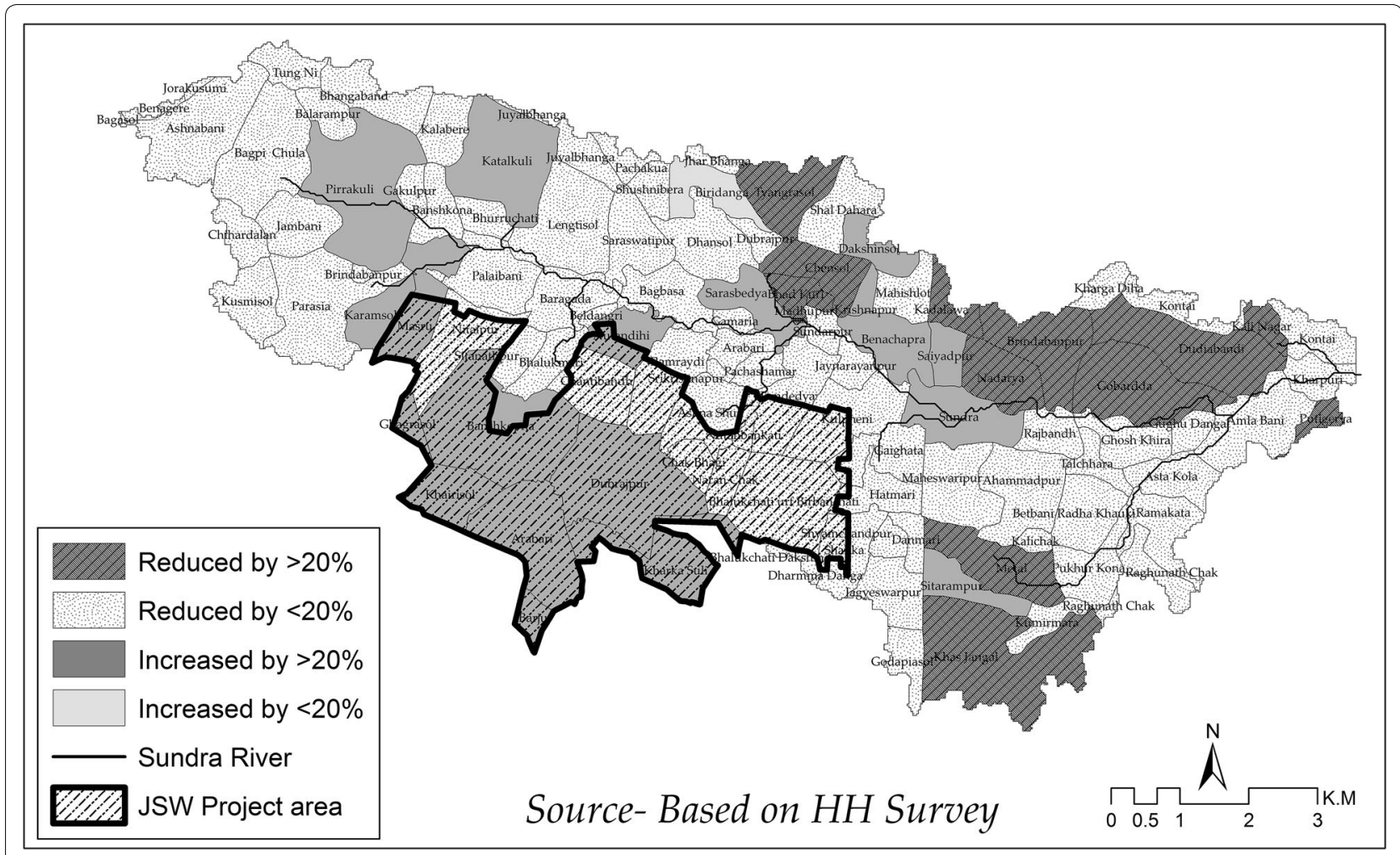

Fig. 3 Showing the changes of marginal workers in between 2001 and 2011

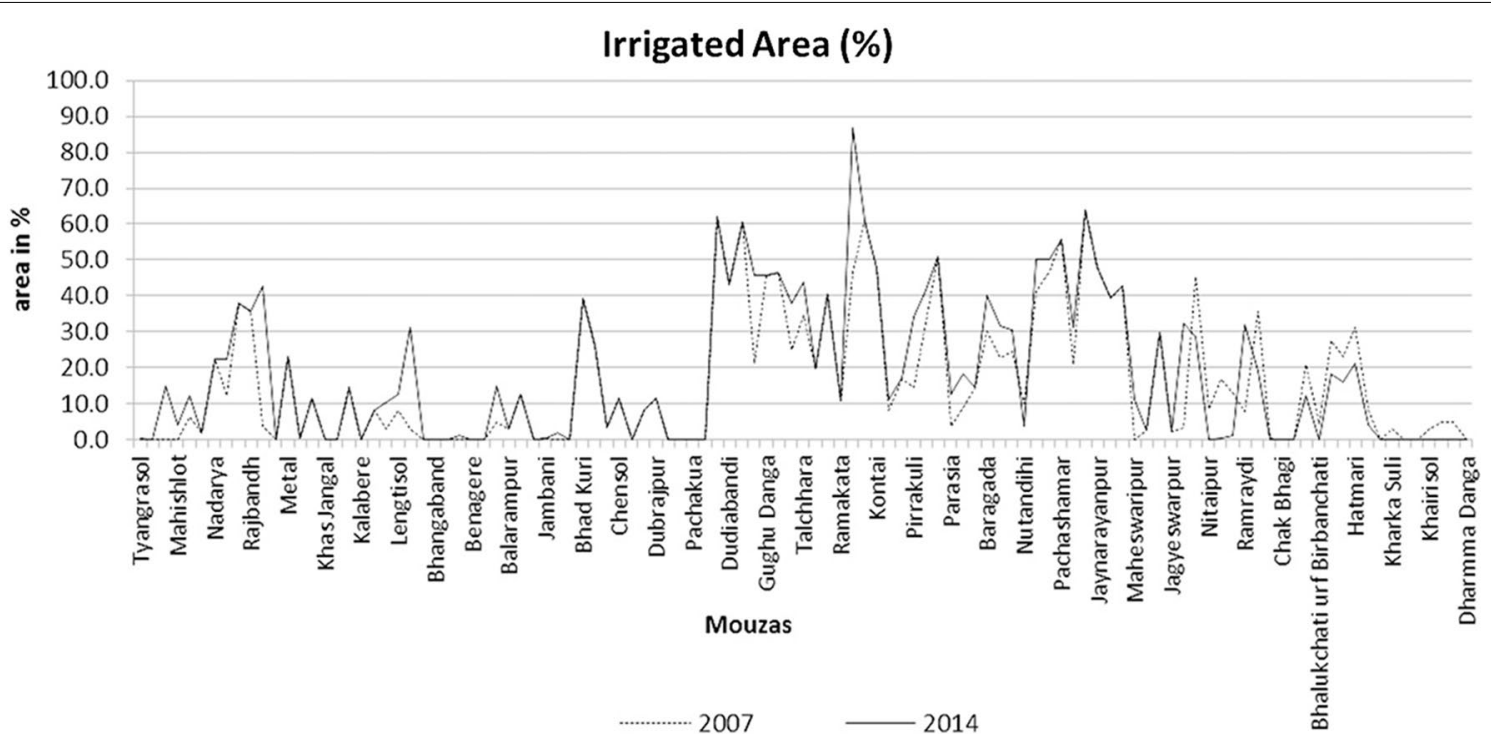

Fig. 4 Showing the distribution of irrigated land in percentage to net sown area

of crop income between pre-acquisition (2007) and postacquisition (2014) condition.

Major reduction has been observed at project site. After land acquisition average crop income is being dropped from $74.1 \%$ in 2007 to $23.2 \%$ in 2014. Major reduction has been observed at Banshkopna, Chantibandh, Kulpheni, Gaighata, Kharka Suli, Ashna Shuli, Arabari and Khairisol mouza. 


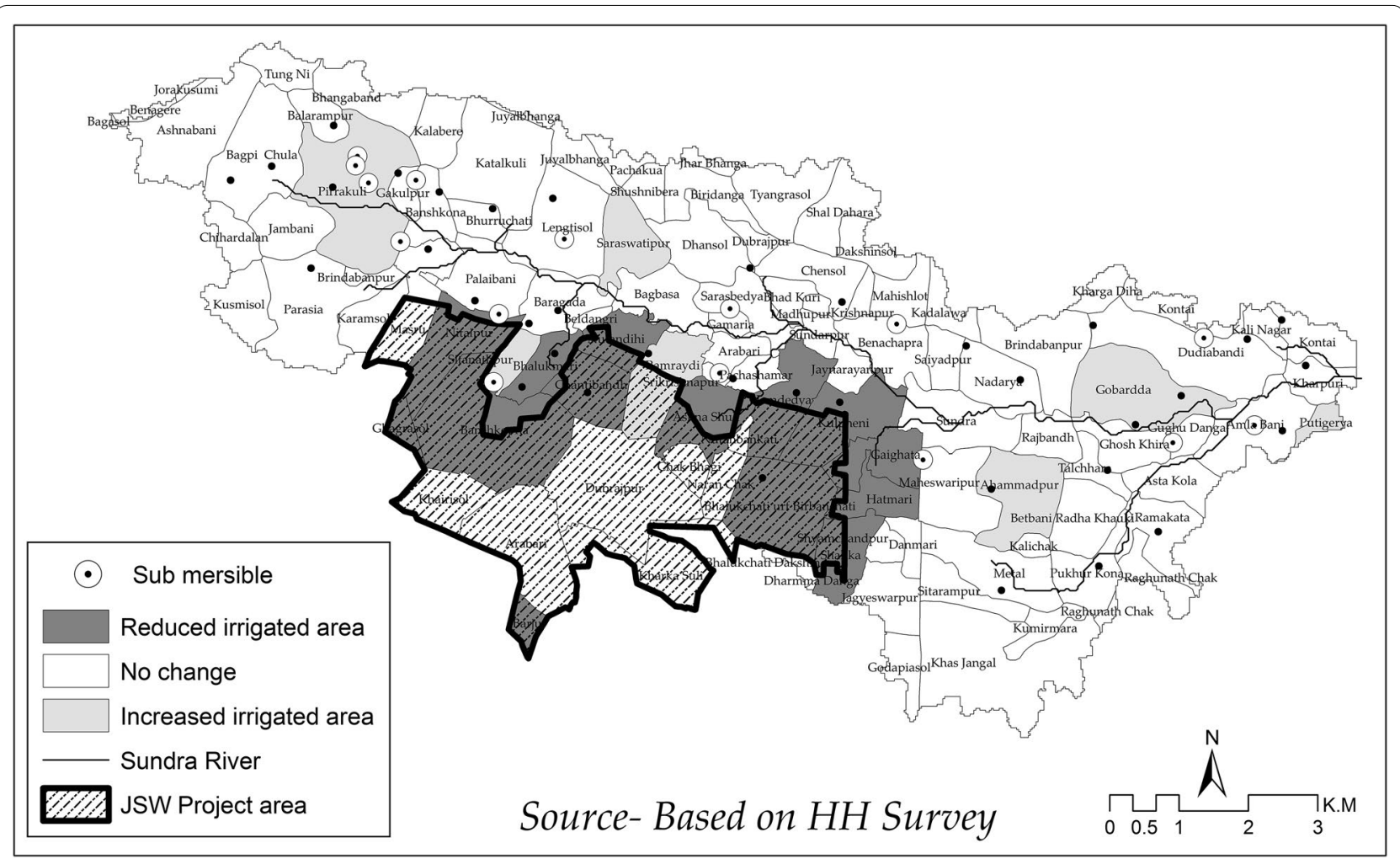

Fig. 5 Showing the changes in irrigated area between 2007 and 2014

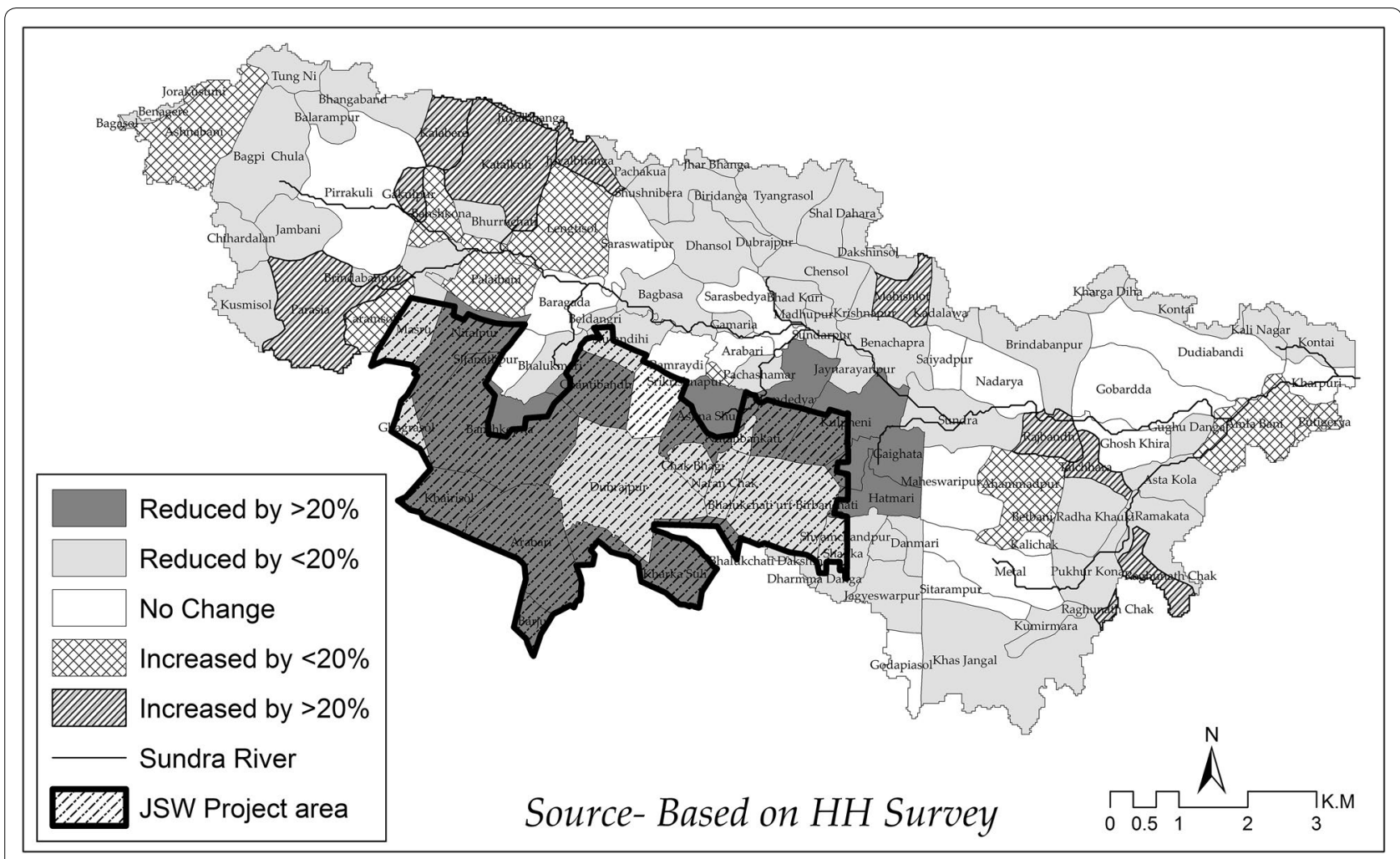

Fig. 6 Showing the changes in crop income in between 2007 and 2014 
Percentage of main cultivators Based on the information available from local BDO office and Census of India, it is observed that average percentage of main cultivator is $33.6 \%$ at study area and it has been observed as $37.4 \%$ in non-project affected mouzas to $18.1 \%$ in project affected mouzas in 2014.

But before acquisition (2007), from available data, main agricultural workers were $35.1 \%$ in non-project affected mouzas and in project affected 22 mouzas it was $28.2 \%$. In 2014 after improve in irrigation facility in non-project mouzas like Bhangaband, Ahammadpur, Saraswatipur, Gobardda, Putigerya, Mahishlot, Kadalawa, Brindabanpur, Rajbandh, Ahammadpur, Metal, Bhangaband, Chensol, Pachakua, Maheswaripur mouza experienced increase in percentage of cultivators by $20-25 \%$ (Fig. 7).

But on the other hand at project site due to land acquisition farm size becomes too small, which is not sufficient to maintain their livelihood. As a consequence, a reverse situation has been obtained here. From Nutandihi, Banshkopna, Chantibandh, Kharka Suli, Nitaipur, Ramraydi, Hatmari and Khairisol mouza more than $30 \%$ of agricultural workers are now being engaged in non-farm activities.

\section{Agricultural composite index ( $\mathrm{Cl}$ ) of development and rank}

On the basis of above indicators followed by Narain et al. 2011, a CI of agricultural development along with ordinal rank and level of development has been calculated for 109 mouzas to compare between the situation in preacquisition (2007) and that in existing condition (2014). From Table 1 in 2007 before acquisition, Dudiabandi mouza was ranked in first place followed by Amla Bani (2nd), Ghosh Khira (3rd), Kharpuri (4th), Bagpi Chula (5th), Rajbandh (6th), Putigerya (7th), Kharka Suli (8th), Asta Kola (9th) and Saiyadpur (10th) mouza and these mouzas were consisted of an average of $64.5 \%$ net sown area, an average $35.1 \%$ of irrigated land, an average $42.1 \%$ of total population was depending on only agricultural activity.

After 5 years of land acquisition (2014) irrigation facility has been improved only at lower catchment and as a consequence more area has come under irrigation and due to delay of JSW project work local people are trying to invest more attention in this sector. From Table 1 it has been observed that in present condition (2014), mouza Rajbandh is ranked first in agricultural development followed by Putigerya (2nd), Dudiabandi (3rd), Kontai (4th), Gobardda

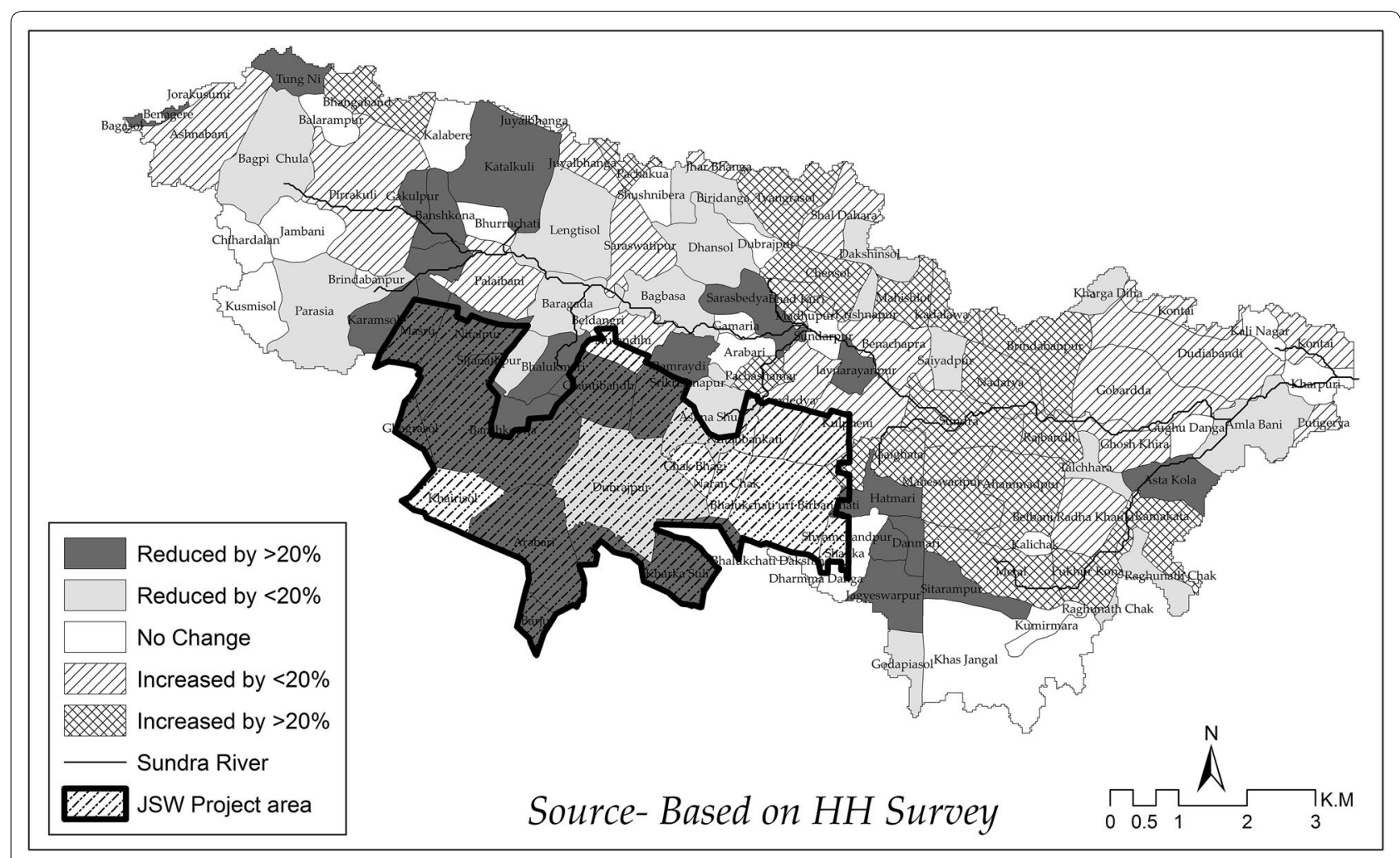

Fig. 7 Showing the changes in main cultivators in between 2001 and 2011 
Table 1 Agricultural composite index (CI) of development and Rank of the mouzas in between 2007 and 2014. Source Author's own calculation

\begin{tabular}{|c|c|c|c|c|c|c|c|c|c|}
\hline \multirow[t]{2}{*}{ SL no. } & \multirow[t]{2}{*}{ Mouza } & \multirow{2}{*}{$\begin{array}{l}\text { Area of mouza } \\
\text { in sq km }\end{array}$} & \multicolumn{3}{|l|}{2007} & \multicolumn{3}{|c|}{2014} & \multirow{2}{*}{$\begin{array}{l}\text { Position gained (+) } \\
\text { or lost }(-) \text { respect } \\
\text { to } 2007 \text { C.I ranking }\end{array}$} \\
\hline & & & $\mathrm{Cl}$ & Rank & $\begin{array}{l}\text { Level of } \\
\text { development }\end{array}$ & $\mathrm{Cl}$ & Rank & $\begin{array}{l}\text { Level of } \\
\text { development }\end{array}$ & \\
\hline 1 & Rajbandh & 0.45 & 0.29 & 6 & High development & 0.28 & 1 & High development & $(+5)$ \\
\hline 2 & Putigerya & 0.98 & 0.31 & 7 & High development & 0.37 & 2 & High development & $(+5)$ \\
\hline 3 & Dudiabandi & 1.74 & 0.13 & 1 & High development & 0.45 & 3 & High development & $(-2)$ \\
\hline 4 & Kontai & 3.55 & 0.39 & 15 & $\begin{array}{l}\text { High middle devel- } \\
\text { opment }\end{array}$ & 0.45 & 4 & High development & $(+11)$ \\
\hline 5 & Gobardda & 2.29 & 0.37 & 12 & High development & 0.45 & 5 & High development & $(+7)$ \\
\hline 6 & Asta Kola & 1.15 & 0.33 & 9 & High development & 0.46 & 6 & High development & $(+3)$ \\
\hline 7 & Maheswaripur & 1.26 & 0.39 & 16 & $\begin{array}{l}\text { High middle devel- } \\
\text { opment }\end{array}$ & 0.48 & 7 & High development & $(+9)$ \\
\hline 8 & Ghosh Khira & 0.55 & 0.28 & 3 & High development & 0.48 & 8 & High development & $(-5)$ \\
\hline 9 & Ahammadpur & 1.38 & 0.39 & 19 & $\begin{array}{l}\text { High middle devel- } \\
\text { opment }\end{array}$ & 0.5 & 9 & High development & $(+10)$ \\
\hline 10 & Amla Bani & 1.67 & 0.26 & 2 & High development & 0.55 & 10 & High development & $(-8)$ \\
\hline 11 & Kharpuri & 0.44 & 0.28 & 4 & High development & 0.55 & 11 & High development & $(-7)$ \\
\hline 12 & Bagpi Chula & 1.71 & 0.29 & 5 & High development & 0.56 & 12 & High development & $(-7)$ \\
\hline 13 & Talchhara & 0.38 & 0.38 & 13 & $\begin{array}{l}\text { High middle devel- } \\
\text { opment }\end{array}$ & 0.56 & 13 & $\begin{array}{l}\text { High middle devel- } \\
\text { opment }\end{array}$ & No change \\
\hline 14 & Madhupur & 0.09 & 0.39 & 18 & $\begin{array}{l}\text { High middle devel- } \\
\text { opment }\end{array}$ & 0.57 & 14 & $\begin{array}{l}\text { High middle devel- } \\
\text { opment }\end{array}$ & $(+4)$ \\
\hline 15 & Baragada & 0.85 & 0.47 & 39 & $\begin{array}{l}\text { Low middle devel- } \\
\text { opment }\end{array}$ & 0.59 & 15 & $\begin{array}{l}\text { High middle devel- } \\
\text { opment }\end{array}$ & $(+24)$ \\
\hline 16 & Sarasbedya & 0.74 & 0.46 & 32 & $\begin{array}{l}\text { High middle devel- } \\
\text { opment }\end{array}$ & 0.59 & 16 & $\begin{array}{l}\text { High middle devel- } \\
\text { opment }\end{array}$ & $(+16)$ \\
\hline 17 & Saiyadpur & 0.47 & 0.34 & 10 & High development & 0.59 & 17 & $\begin{array}{l}\text { High middle devel- } \\
\text { opment }\end{array}$ & $(-7)$ \\
\hline 18 & Kadalawa & 1.12 & 0.36 & 11 & High development & 0.59 & 18 & $\begin{array}{l}\text { High middle devel- } \\
\text { opment }\end{array}$ & $(-7)$ \\
\hline 19 & Sitanathpur & 0.47 & 0.5 & 57 & $\begin{array}{l}\text { Low middle devel- } \\
\text { opment }\end{array}$ & 0.6 & 19 & $\begin{array}{l}\text { High middle devel- } \\
\text { opment }\end{array}$ & $(+38)$ \\
\hline 20 & Dhansol & 1.09 & 0.48 & 48 & $\begin{array}{l}\text { Low middle devel- } \\
\text { opment }\end{array}$ & 0.6 & 20 & $\begin{array}{l}\text { High middle devel- } \\
\text { opment }\end{array}$ & $(+28)$ \\
\hline 21 & Raghunath Chak & 1.01 & 0.41 & 20 & $\begin{array}{l}\text { High middle devel- } \\
\text { opment }\end{array}$ & 0.6 & 21 & $\begin{array}{l}\text { High middle devel- } \\
\text { opment }\end{array}$ & $(-1)$ \\
\hline 22 & Gakulpur & 0.26 & 0.47 & 36 & $\begin{array}{l}\text { Low middle devel- } \\
\text { opment }\end{array}$ & 0.61 & 22 & $\begin{array}{l}\text { High middle devel- } \\
\text { opment }\end{array}$ & $(+14)$ \\
\hline 23 & Saraswatipur & 0.9 & 0.46 & 31 & $\begin{array}{l}\text { High middle devel- } \\
\text { opment }\end{array}$ & 0.61 & 23 & $\begin{array}{l}\text { High middle devel- } \\
\text { opment }\end{array}$ & $(+8)$ \\
\hline 24 & Mahishlot & 0.58 & 0.6 & 81 & Low development & 0.61 & 24 & $\begin{array}{l}\text { High middle devel- } \\
\text { opment }\end{array}$ & $(+57)$ \\
\hline 25 & Nadarya & 0.93 & 0.48 & 47 & $\begin{array}{l}\text { Low middle devel- } \\
\text { opment }\end{array}$ & 0.61 & 25 & $\begin{array}{l}\text { High middle devel- } \\
\text { opment }\end{array}$ & $(+22)$ \\
\hline 26 & Tyangrasol & 3.03 & 0.51 & 60 & $\begin{array}{l}\text { Low middle devel- } \\
\text { opment }\end{array}$ & 0.59 & 26 & $\begin{array}{l}\text { High middle devel- } \\
\text { opment }\end{array}$ & $(+34)$ \\
\hline 27 & Brindabanpur & 1.49 & 0.38 & 14 & $\begin{array}{l}\text { High middle devel- } \\
\text { opment }\end{array}$ & 0.62 & 27 & $\begin{array}{l}\text { High middle devel- } \\
\text { opment }\end{array}$ & $(-13)$ \\
\hline 28 & Dakshinsol & 0.72 & 0.56 & 74 & $\begin{array}{l}\text { Low middle devel- } \\
\text { opment }\end{array}$ & 0.62 & 28 & $\begin{array}{l}\text { High middle devel- } \\
\text { opment }\end{array}$ & $(+46)$ \\
\hline 29 & Lengtisol & 1.68 & 0.47 & 43 & $\begin{array}{l}\text { Low middle devel- } \\
\text { opment }\end{array}$ & 0.62 & 29 & $\begin{array}{l}\text { High middle devel- } \\
\text { opment }\end{array}$ & $(+14)$ \\
\hline 30 & Sundra & 1 & 0.48 & 46 & $\begin{array}{l}\text { Low middle devel- } \\
\text { opment }\end{array}$ & 0.62 & 30 & $\begin{array}{l}\text { High middle devel- } \\
\text { opment }\end{array}$ & $(+16)$ \\
\hline
\end{tabular}


Table 1 continued

\begin{tabular}{|c|c|c|c|c|c|c|c|c|c|}
\hline \multirow[t]{2}{*}{ SL no. } & \multirow[t]{2}{*}{ Mouza } & \multirow{2}{*}{$\begin{array}{l}\text { Area of mouza } \\
\text { in sq km }\end{array}$} & \multicolumn{3}{|c|}{2007} & \multicolumn{3}{|c|}{2014} & \multirow{2}{*}{$\begin{array}{l}\text { Position gained (+) } \\
\text { or lost }(-) \text { respect } \\
\text { to } 2007 \text { C.I ranking }\end{array}$} \\
\hline & & & $\mathrm{Cl}$ & Rank & $\begin{array}{l}\text { Level of } \\
\text { development }\end{array}$ & $\mathrm{Cl}$ & Rank & $\begin{array}{l}\text { Level of } \\
\text { development }\end{array}$ & \\
\hline 31 & Ramraydi & 1.12 & 0.47 & 42 & $\begin{array}{l}\text { Low middle devel- } \\
\text { opment }\end{array}$ & 0.63 & 31 & $\begin{array}{l}\text { High middle devel- } \\
\text { opment }\end{array}$ & $(+11)$ \\
\hline 32 & Srikrishnapur & 0.18 & 0.49 & 53 & $\begin{array}{l}\text { Low middle devel- } \\
\text { opment }\end{array}$ & 0.63 & 32 & $\begin{array}{l}\text { High middle devel- } \\
\text { opment }\end{array}$ & $(+21)$ \\
\hline 33 & Nutandihi & 0.71 & 0.46 & 29 & $\begin{array}{l}\text { High middle devel- } \\
\text { opment }\end{array}$ & 0.63 & 33 & $\begin{array}{l}\text { High middle devel- } \\
\text { opment }\end{array}$ & $(-4)$ \\
\hline 34 & Karamsol & 0.54 & 0.47 & 38 & $\begin{array}{l}\text { Low middle devel- } \\
\text { opment }\end{array}$ & 0.64 & 34 & $\begin{array}{l}\text { High middle devel- } \\
\text { opment }\end{array}$ & $(+4)$ \\
\hline 35 & Bagbasa & 1.07 & 0.45 & 27 & $\begin{array}{l}\text { High middle devel- } \\
\text { opment }\end{array}$ & 0.65 & 35 & $\begin{array}{l}\text { High middle devel- } \\
\text { opment }\end{array}$ & $(-8)$ \\
\hline 36 & Palaibani & 1.3 & 0.48 & 44 & $\begin{array}{l}\text { Low middle devel- } \\
\text { opment }\end{array}$ & 0.66 & 36 & $\begin{array}{l}\text { High middle devel- } \\
\text { opment }\end{array}$ & $(+8)$ \\
\hline 37 & Shalika & 1.02 & 0.48 & 49 & $\begin{array}{l}\text { Low middle devel- } \\
\text { opment }\end{array}$ & 0.66 & 37 & $\begin{array}{l}\text { High middle devel- } \\
\text { opment }\end{array}$ & $(+12)$ \\
\hline 38 & Gaighata & 0.86 & 0.47 & 41 & $\begin{array}{l}\text { Low middle devel- } \\
\text { opment }\end{array}$ & 0.66 & 38 & $\begin{array}{l}\text { High middle devel- } \\
\text { opment }\end{array}$ & $(+3)$ \\
\hline 39 & Pirrakuli & 2.59 & 0.47 & 37 & $\begin{array}{l}\text { Low middle devel- } \\
\text { opment }\end{array}$ & 0.67 & 39 & $\begin{array}{l}\text { High middle devel- } \\
\text { opment }\end{array}$ & $(-2)$ \\
\hline 40 & Banshkona & 0.7 & 0.45 & 22 & $\begin{array}{l}\text { High middle devel- } \\
\text { opment }\end{array}$ & 0.67 & 40 & $\begin{array}{l}\text { High middle devel- } \\
\text { opment }\end{array}$ & $(-18)$ \\
\hline 41 & $\begin{array}{l}\text { Betbani Radha } \\
\text { Khauki }\end{array}$ & 0.91 & 0.5 & 58 & $\begin{array}{l}\text { Low middle devel- } \\
\text { opment }\end{array}$ & 0.67 & 41 & $\begin{array}{l}\text { High middle devel- } \\
\text { opment }\end{array}$ & $(+17)$ \\
\hline 42 & Shal Dahara & 1.56 & 0.55 & 71 & $\begin{array}{l}\text { Low middle devel- } \\
\text { opment }\end{array}$ & 0.68 & 42 & $\begin{array}{l}\text { High middle devel- } \\
\text { opment }\end{array}$ & $(+29)$ \\
\hline 43 & Ashnabani & 2.59 & 0.51 & 62 & $\begin{array}{l}\text { Low middle devel- } \\
\text { opment }\end{array}$ & 0.68 & 43 & $\begin{array}{l}\text { High middle devel- } \\
\text { opment }\end{array}$ & $(+19)$ \\
\hline 44 & Krishnapur & 0.17 & 0.53 & 68 & $\begin{array}{l}\text { Low middle devel- } \\
\text { opment }\end{array}$ & 0.68 & 44 & $\begin{array}{l}\text { High middle devel- } \\
\text { opment }\end{array}$ & $(+24)$ \\
\hline 45 & Dubrajpur & 2.59 & NA & NA & NA & 0.69 & 45 & $\begin{array}{l}\text { High middle devel- } \\
\text { opment }\end{array}$ & NA \\
\hline 46 & Benachapra & 0.91 & 0.51 & 61 & $\begin{array}{l}\text { Low middle devel- } \\
\text { opment }\end{array}$ & 0.69 & 46 & $\begin{array}{l}\text { Low middle devel- } \\
\text { opment }\end{array}$ & $(+15)$ \\
\hline 47 & Pukhur Kona & 0.74 & 0.57 & 76 & Low development & 0.7 & 47 & $\begin{array}{l}\text { Low middle devel- } \\
\text { opment }\end{array}$ & $(+29)$ \\
\hline 48 & Banshkopna & 2.87 & 0.45 & 24 & $\begin{array}{l}\text { High middle devel- } \\
\text { opment }\end{array}$ & 0.72 & 48 & $\begin{array}{l}\text { Low middle devel- } \\
\text { opment }\end{array}$ & $(-24)$ \\
\hline 49 & Kali Nagar & 0.28 & 0.51 & 65 & $\begin{array}{l}\text { Low middle devel- } \\
\text { opment }\end{array}$ & 0.72 & 49 & $\begin{array}{l}\text { Low middle devel- } \\
\text { opment }\end{array}$ & $(+16)$ \\
\hline 50 & Masru & 1.03 & 0.45 & 26 & $\begin{array}{l}\text { High middle devel- } \\
\text { opment }\end{array}$ & 0.73 & 50 & $\begin{array}{l}\text { Low middle devel- } \\
\text { opment }\end{array}$ & $(-24)$ \\
\hline 51 & Chantibandh & 0.92 & 0.45 & 23 & $\begin{array}{l}\text { High middle devel- } \\
\text { opment }\end{array}$ & 0.73 & 51 & $\begin{array}{l}\text { Low middle devel- } \\
\text { opment }\end{array}$ & $(-28)$ \\
\hline 52 & Bhad Kuri & 0.34 & 0.56 & 75 & Low development & 0.73 & 52 & $\begin{array}{l}\text { Low middle devel- } \\
\text { opment }\end{array}$ & $(+23)$ \\
\hline 53 & Ashna Shuli & 1.06 & 0.46 & 30 & $\begin{array}{l}\text { High middle devel- } \\
\text { opment }\end{array}$ & 0.74 & 53 & $\begin{array}{l}\text { Low middle devel- } \\
\text { opment }\end{array}$ & $(-23)$ \\
\hline 54 & Arabari & 1.94 & 0.51 & 64 & $\begin{array}{l}\text { Low middle devel- } \\
\text { opment }\end{array}$ & 0.74 & 54 & $\begin{array}{l}\text { Low middle devel- } \\
\text { opment }\end{array}$ & $(+10)$ \\
\hline 55 & Sitarampur & 0.63 & 0.57 & 79 & Low development & 0.74 & 55 & $\begin{array}{l}\text { Low middle devel- } \\
\text { opment }\end{array}$ & $(+24)$ \\
\hline 56 & Kulpheni & 1.35 & 0.39 & 17 & $\begin{array}{l}\text { High middle devel- } \\
\text { opment }\end{array}$ & 0.76 & 56 & $\begin{array}{l}\text { Low middle devel- } \\
\text { opment }\end{array}$ & $(-39)$ \\
\hline 57 & Kalabere & 0.7 & NA & NA & NA & 0.76 & 57 & $\begin{array}{l}\text { Low middle devel- } \\
\text { opment }\end{array}$ & NA \\
\hline
\end{tabular}


Table 1 continued

\begin{tabular}{|c|c|c|c|c|c|c|c|c|c|}
\hline \multirow[t]{2}{*}{ SL no. } & \multirow[t]{2}{*}{ Mouza } & \multirow{2}{*}{$\begin{array}{l}\text { Area of mouza } \\
\text { in sq km }\end{array}$} & \multicolumn{3}{|c|}{2007} & \multicolumn{3}{|c|}{2014} & \multirow{2}{*}{$\begin{array}{l}\text { Position gained }(+) \\
\text { or lost(-) respect } \\
\text { to } 2007 \text { C.I ranking }\end{array}$} \\
\hline & & & $\mathrm{Cl}$ & Rank & $\begin{array}{l}\text { Level of } \\
\text { development }\end{array}$ & $\mathrm{Cl}$ & Rank & $\begin{array}{l}\text { Level of } \\
\text { development }\end{array}$ & \\
\hline 58 & Biridanga & 0.73 & 0.49 & 52 & $\begin{array}{l}\text { Low middle devel- } \\
\text { opment }\end{array}$ & 0.76 & 58 & $\begin{array}{l}\text { Low middle devel- } \\
\text { opment }\end{array}$ & $(-6)$ \\
\hline 59 & Jagyeswarpur & 1.12 & 0.46 & 33 & $\begin{array}{l}\text { High middle devel- } \\
\text { opment }\end{array}$ & 0.77 & 59 & $\begin{array}{l}\text { Low middle devel- } \\
\text { opment }\end{array}$ & $(-26)$ \\
\hline 60 & Ramakata & 1.35 & 0.52 & 66 & $\begin{array}{l}\text { Low middle devel- } \\
\text { opment }\end{array}$ & 0.77 & 60 & $\begin{array}{l}\text { Low middle devel- } \\
\text { opment }\end{array}$ & $(+6)$ \\
\hline 61 & Sundarpur & 0.34 & NA & NA & NA & 0.77 & 61 & $\begin{array}{l}\text { Low middle devel- } \\
\text { opment }\end{array}$ & NA \\
\hline 62 & Nitaipur & 1.21 & 0.49 & 51 & $\begin{array}{l}\text { Low middle devel- } \\
\text { opment }\end{array}$ & 0.78 & 62 & $\begin{array}{l}\text { Low middle devel- } \\
\text { opment }\end{array}$ & $(-11)$ \\
\hline 63 & Khas Jangal & 7.5 & 0.45 & 21 & $\begin{array}{l}\text { High middle devel- } \\
\text { opment }\end{array}$ & 0.78 & 63 & $\begin{array}{l}\text { Low middle devel- } \\
\text { opment }\end{array}$ & $(-42)$ \\
\hline 64 & Bhangaband & 2.67 & 0.57 & 78 & Low development & 0.78 & 64 & $\begin{array}{l}\text { Low middle devel- } \\
\text { opment }\end{array}$ & $(+14)$ \\
\hline 65 & Barju & 0.99 & 0.47 & 40 & $\begin{array}{l}\text { Low middle devel- } \\
\text { opment }\end{array}$ & 0.78 & 65 & $\begin{array}{l}\text { Low middle devel- } \\
\text { opment }\end{array}$ & $(-25)$ \\
\hline 66 & Hatmari & 0.61 & 0.49 & 55 & $\begin{array}{l}\text { Low middle devel- } \\
\text { opment }\end{array}$ & 0.78 & 66 & $\begin{array}{l}\text { Low middle devel- } \\
\text { opment }\end{array}$ & $(-11)$ \\
\hline 67 & Juyalbhanga & 2.35 & 0.5 & 59 & $\begin{array}{l}\text { Low middle devel- } \\
\text { opment }\end{array}$ & 0.79 & 67 & $\begin{array}{l}\text { Low middle devel- } \\
\text { opment }\end{array}$ & $(-8)$ \\
\hline 68 & Tung Ni & 1.89 & 0.62 & 82 & Low development & 0.79 & 68 & $\begin{array}{l}\text { Low middle devel- } \\
\text { opment }\end{array}$ & $(+14)$ \\
\hline 69 & Durgadaspur & 0.05 & 0.49 & 50 & $\begin{array}{l}\text { Low middle devel- } \\
\text { opment }\end{array}$ & 0.79 & 69 & $\begin{array}{l}\text { Low middle devel- } \\
\text { opment }\end{array}$ & $(-19)$ \\
\hline 70 & Katalkuli & 1.97 & 0.51 & 63 & $\begin{array}{l}\text { Low middle devel- } \\
\text { opment }\end{array}$ & 0.8 & 70 & $\begin{array}{l}\text { Low middle devel- } \\
\text { opment }\end{array}$ & $(-7)$ \\
\hline 71 & Shyamchandpur & 0.18 & 0.58 & 80 & Low development & 0.8 & 71 & $\begin{array}{l}\text { Low middle devel- } \\
\text { opment }\end{array}$ & $(+9)$ \\
\hline 72 & Pachashamar & 0.33 & NA & NA & NA & 0.8 & 72 & $\begin{array}{l}\text { Low middle devel- } \\
\text { opment }\end{array}$ & NA \\
\hline 73 & Kharka Suli & 1.58 & 0.31 & 8 & High development & 0.81 & 73 & $\begin{array}{l}\text { Low middle devel- } \\
\text { opment }\end{array}$ & $(-65)$ \\
\hline 74 & Jamdedya & 1.12 & 0.49 & 56 & $\begin{array}{l}\text { Low middle devel- } \\
\text { opment }\end{array}$ & 0.8 & 74 & $\begin{array}{l}\text { Low middle devel- } \\
\text { opment }\end{array}$ & $(-18)$ \\
\hline 75 & Metal & 1.19 & 0.55 & 72 & $\begin{array}{l}\text { Low middle devel- } \\
\text { opment }\end{array}$ & 0.81 & 75 & $\begin{array}{l}\text { Low middle devel- } \\
\text { opment }\end{array}$ & $(-3)$ \\
\hline 76 & Parasia & 2.01 & 0.45 & 25 & $\begin{array}{l}\text { High middle devel- } \\
\text { opment }\end{array}$ & 0.82 & 76 & $\begin{array}{l}\text { Low middle devel- } \\
\text { opment }\end{array}$ & $(-51)$ \\
\hline 77 & Jaynarayanpur & 0.37 & 0.47 & 35 & $\begin{array}{l}\text { Low middle devel- } \\
\text { opment }\end{array}$ & 0.82 & 77 & Low development & $(-42)$ \\
\hline 78 & Kalichak & 0.13 & NA & NA & NA & 0.83 & 78 & Low development & NA \\
\hline 79 & Godapiasol & 2.33 & 0.66 & 84 & Low development & 0.84 & 79 & Low development & $(+5)$ \\
\hline 80 & Arabari & 0.26 & 0.46 & 28 & $\begin{array}{l}\text { High middle devel- } \\
\text { opment }\end{array}$ & 0.84 & 80 & Low development & $(-52)$ \\
\hline 81 & Kharga Diha & 1.52 & 0.46 & 34 & $\begin{array}{l}\text { High middle devel- } \\
\text { opment }\end{array}$ & 0.84 & 81 & Low development & $(-47)$ \\
\hline 82 & Bhalukmari & 0.49 & 0.53 & 69 & $\begin{array}{l}\text { Low middle devel- } \\
\text { opment }\end{array}$ & 0.84 & 82 & Low development & $(-13)$ \\
\hline 83 & Chensol & 0.83 & 0.52 & 67 & $\begin{array}{l}\text { Low middle devel- } \\
\text { opment }\end{array}$ & 0.86 & 83 & Low development & $(-16)$ \\
\hline 84 & Benagere & 1.98 & 0.48 & 45 & $\begin{array}{l}\text { Low middle devel- } \\
\text { opment }\end{array}$ & 0.87 & 84 & Low development & $(-39)$ \\
\hline 85 & Ghagrasol & 1.26 & 0.54 & 70 & $\begin{array}{l}\text { Low middle devel- } \\
\text { opment }\end{array}$ & 0.87 & 85 & Low development & $(-15)$ \\
\hline
\end{tabular}


Table 1 continued

\begin{tabular}{|c|c|c|c|c|c|c|c|c|c|}
\hline \multirow[t]{2}{*}{ SL no. } & \multirow[t]{2}{*}{ Mouza } & \multirow{2}{*}{$\begin{array}{l}\text { Area of mouza } \\
\text { in sq km }\end{array}$} & \multicolumn{3}{|c|}{2007} & \multicolumn{3}{|c|}{2014} & \multirow{2}{*}{$\begin{array}{l}\text { Position gained (+) } \\
\text { or lost(-) respect } \\
\text { to } 2007 \text { C.I ranking }\end{array}$} \\
\hline & & & $\mathrm{Cl}$ & Rank & $\begin{array}{l}\text { Level of } \\
\text { development }\end{array}$ & $\mathrm{Cl}$ & Rank & $\begin{array}{l}\text { Level of } \\
\text { development }\end{array}$ & \\
\hline 86 & Danmari & 0.25 & 0.62 & 83 & Low development & 0.88 & 86 & Low development & $(-3)$ \\
\hline 87 & Jhar Bhanga & 2.05 & 0.71 & 85 & Low development & 0.91 & 87 & Low development & $(-2)$ \\
\hline 88 & Khairisol & 1.0 & 0.49 & 54 & $\begin{array}{l}\text { Low middle devel- } \\
\text { opment }\end{array}$ & 0.91 & 88 & Low development & $(-34)$ \\
\hline 89 & Pachakua & 3.18 & 0.56 & 73 & $\begin{array}{l}\text { Low middle devel- } \\
\text { opment }\end{array}$ & 0.92 & 89 & Low development & $(-16)$ \\
\hline 90 & Jorakusumi & 1.68 & 0.57 & 77 & Low development & 0.94 & 90 & Low development & $(-13)$ \\
\hline 91 & Gamaria & 0.26 & NA & NA & NA & NA & NA & NA & NA \\
\hline 92 & Kumirmara & 0.41 & NA & NA & NA & NA & NA & NA & NA \\
\hline 93 & Bhurruchati & 0.35 & NA & NA & NA & NA & NA & NA & NA \\
\hline 94 & Bagasol & 0.39 & NA & NA & NA & NA & NA & NA & NA \\
\hline 95 & Balarampur & 0.37 & NA & NA & NA & NA & NA & NA & NA \\
\hline 96 & Chihardalan & 1.13 & NA & NA & NA & NA & NA & NA & NA \\
\hline 97 & Jambani & 0.82 & NA & NA & NA & NA & NA & NA & NA \\
\hline 98 & Brindabanpur & 0.08 & NA & NA & NA & NA & NA & NA & NA \\
\hline 99 & Kusmisol & 0.8 & NA & NA & NA & NA & NA & NA & NA \\
\hline 100 & Shushnibera & 0.48 & NA & NA & NA & NA & NA & NA & NA \\
\hline 101 & Gughu Danga & 0.24 & NA & NA & NA & NA & NA & NA & NA \\
\hline 102 & Beldangri & 0.08 & NA & NA & NA & NA & NA & NA & NA \\
\hline 103 & Nutanbankati & 1.12 & NA & NA & NA & NA & NA & NA & NA \\
\hline 104 & Chak Bhagi & 0.33 & NA & NA & NA & NA & NA & NA & NA \\
\hline 105 & Naran Chak & 0.4 & NA & NA & NA & NA & NA & NA & NA \\
\hline 106 & $\begin{array}{c}\text { Bhalukchati urf } \\
\text { Birbanchati }\end{array}$ & 1.71 & NA & NA & NA & NA & NA & NA & NA \\
\hline 107 & $\begin{array}{l}\text { Bhalukchati } \\
\text { Dakshin }\end{array}$ & 0.6 & NA & NA & NA & NA & NA & NA & NA \\
\hline 108 & Dubrajpur & 0.2 & NA & NA & NA & NA & NA & NA & NA \\
\hline 109 & Dharmma Danga & 0.41 & NA & NA & NA & NA & NA & NA & NA \\
\hline
\end{tabular}

(5th), Asta Kola (6th), Maheswaripur (7th), Ghosh Khira (8th), Ahammadpur (9th), Amla Bani (10th) mouza. These mouzas are located at the lower catchment of Sundra basin outside the project affected area. These mouzas highly depend on agricultural activity due to availability of good quality of agricultural land and fertile soil. On an average $69.1 \%$ of total area is net sown area. More than $45.8 \%$ area has irrigation facility. On an average, $59.6 \%$ of total population is engaged in agricultural activity.

A dramatic change in mouzas' ordinal rank has been observed among the projected affected mouzas. Most of the project affected mouzas have been retreated from their position, from those of 2007 (Table 1). Before land acquisition (2007) Kharka Suli, Kulpheni, Chantibandh, Banshkopna and Masru mouza ranked by 8th, 17th, 23rd, 24th and 26th respectively but after acquisition in 2014 these mouzas are now standing on 73rd, 56th, 51st, 48th and 50th position respectively.
A cursory look at Column of CI of Table 1 in 2014 reveals that the $\mathrm{CI}$ of agricultural development varies from 0.28 to 0.94 that indicates a greatest regional disparity exiting in agricultural development. From the above discussion it may be concluded that the lower catchment is in relatively advanced stage with comparison of upper catchment. Local people of the project affected mouzas are in severe condition. So a proper planning is needed immediately to reduce these regional disparities in agricultural sector.

\section{Relative share of population under different level of agricultural development}

Tables 2 and 3 present the number of mouzas lying in different levels of development on basis of the measure of development (Eq. 1.4) in agricultural sector in existing condition (2014) and that was in 2007, before acquisition. 
Table 2 Number of mouzas with percentage of area and population under different level of agricultural development in 2014. Source Author's own calculation

\begin{tabular}{|c|c|c|c|c|}
\hline Level of development & $\begin{array}{l}\text { Number } \\
\text { of mouzas }\end{array}$ & Name of mouzas & $\begin{array}{l}\% \text { of total } \\
\text { population }\end{array}$ & $\begin{array}{l}\% \text { of total } \\
\text { area }\end{array}$ \\
\hline \multicolumn{5}{|c|}{ Agricultural level of development } \\
\hline High & 12 & $\begin{array}{l}\text { Rajbandh, Ahammadpur, Bagpi Chula, Dudiabandi, Ghosh Khira, Gobardda, } \\
\text { Amla Bani, Asta Kola, Putigerya, Kharpuri, Kontai, Maheswaripur }\end{array}$ & 17.46 & 16.92 \\
\hline High middle & 33 & $\begin{array}{l}\text { Tyangrasol, Shal Dahara, Dakshinsol, Mahishlot, Kadalawa, Brindabanpur, } \\
\text { Nadarya, Saiyadpur, Gakulpur, Lengtisol, Saraswatipur, Ashnabani, Mad- } \\
\text { hupur, Krishnapur, Dhansol, Dubrajpur, Talchhara, Betbani Radha Khauki, } \\
\text { Raghunath Chak, Banshkona, Pirrakuli, Palaibani, Karamsol, Baragada, Bag- } \\
\text { basa, Nutandihi, Srikrishnapur, Sarasbedya, Sundra, Sitanathpur, Ramraydi, } \\
\text { Gaighata, Shalika }\end{array}$ & 34.46 & 37.41 \\
\hline Low middle & 31 & $\begin{array}{l}\text { Metal, Sitarampur, Khas Jangal, Kalabere, Katalkuli, Juyalbhanga, Bhangaband, } \\
\text { Tung Ni, Bhad Kuri, Biridanga, Kali Nagar, Pukhur Kona, Ramakata, Dur- } \\
\text { gadaspur, Parasia, Masru, Pachashamar, Sundarpur, Benachapra, Shyam- } \\
\text { chandpur, Jagyeswarpur, Nitaipur, Banshkopna, Chantibandh, Ashna Shuli, } \\
\text { Jamdedya, Kulpheni, Hatmari, Kharka Suli, Arabari, Barju }\end{array}$ & 30.08 & 39.38 \\
\hline Low & 14 & $\begin{array}{l}\text { Kalichak, Godapiasol, Jorakusumi, Benagere, Chensol, Jhar Bhanga, Pachakua, } \\
\text { Kharga Diha, Arabari, Jaynarayanpur, Danmari, Bhalukmari, Khairisol, Gha- } \\
\text { grasol }\end{array}$ & 17.99 & 6.29 \\
\hline
\end{tabular}

It has been concluded that in existing condition (2014) (Fig. 8) only 12 mouzas are found to be in high level of development category in agricultural sector that covers $16.9 \%$ of total area and $17.4 \%$ of total population. Before acquisition (2007) (Fig. 9) 14.6\% of total population covering $15.8 \%$ area is characterised by high level of development. High middle level development category covers $34.4 \%$ of total population in 33 mouzas spread over $37.4 \%$ of total area in 2014. But in 2007, there was $26.1 \%$ of total population in 22 mouzas. 45 mouzas are found to be in low and low middle development category in 2014 that covers $45.8 \%$ of the total area and $48.1 \%$ of population, but before acquisition (2007) total 51 mouzas were observed in this category that covered $52.1 \%$ of total area and $59.2 \%$ of total population.

No mouza at project site has been observed in high development category after acquisition. At project site four mouzas namely Nutandihi, Ramraydi, Gaighata and Shalika are found to be in high middle level of agricultural development category in 2014 (Table 4). These mouzas cover $19.2 \%$ of project affected area and $22.04 \%$ of project affected population. Before acquisition in 2007 six project affected mouzas were found in high middle level development category and these mouzas covered by $39.4 \%$ of total project affected population and $46.4 \%$ of project affected area. In low level

Table 3 Number of mouzas with percentage of area and population under different level of agricultural development in 2007. Source Author's own calculation

\begin{tabular}{|c|c|c|c|c|}
\hline Stages of development & $\begin{array}{l}\text { Number } \\
\text { of mouzas }\end{array}$ & Name of mouzas & $\begin{array}{l}\% \text { of total } \\
\text { population }\end{array}$ & $\begin{array}{l}\% \text { of total } \\
\text { area }\end{array}$ \\
\hline \multicolumn{5}{|c|}{ Agricultural level of development } \\
\hline High & 12 & $\begin{array}{l}\text { Kadalawa, Saiyadpur, Rajbandh, Bagpi Chula, Dudiabandi, Ghosh Khira, Gob- } \\
\text { ardda, Amla Bani, Asta Kola, Putigerya, Kharpuri, Kharka Suli }\end{array}$ & 14.62 & 15.80 \\
\hline High middle & 22 & $\begin{array}{l}\text { Brindabanpur, Ahammadpur, Khas Jangal, Saraswatipur, Madhupur, Kharga } \\
\text { Diha, Talchhara, Kontai, Raghunath Chak, Banshkona, Parasia, Masru, Bagbasa, } \\
\text { Nutandihi, Arabari, Sarasbedya, Maheswaripur, Jagyeswarpur, Banshkopna, } \\
\text { Chantibandh, Ashna Shuli, Kulpheni }\end{array}$ & 26.11 & 32.07 \\
\hline Low middle & 40 & $\begin{array}{l}\text { Tyangrasol, Shal Dahara, Dakshinsol, Nadarya, Metal, Gakulpur, Katalkuli, Lengtisol, } \\
\text { Juyalbhanga, Benagere, Ashnabani, Krishnapur, Chensol, Dhansol, Biridanga, } \\
\text { Pachakua, Kali Nagar, Betbani Radha Khauki, Ramakata, Pirrakuli, Palaibani, } \\
\text { Durgadaspur, Karamsol, Baragada, Srikrishnapur, Jaynarayanpur, Benachapra, } \\
\text { Sundra, Sitanathpur, Bhalukmari, Nitaipur, Ramraydi, Jamdedya, Gaighata, } \\
\text { Hatmari, Arabari, Khairisol, Barju, Ghagrasol, Shalika }\end{array}$ & 46.74 & 45.51 \\
\hline Low & 11 & $\begin{array}{l}\text { Mahishlot, Sitarampur, Godapiasol, Bhangaband, Tung Ni, Jorakusumi, Bhad Kuri, } \\
\text { Jhar Bhanga, Danmari, Shyamchandpur, Pukhur Kona }\end{array}$ & 12.52 & 6.63 \\
\hline
\end{tabular}




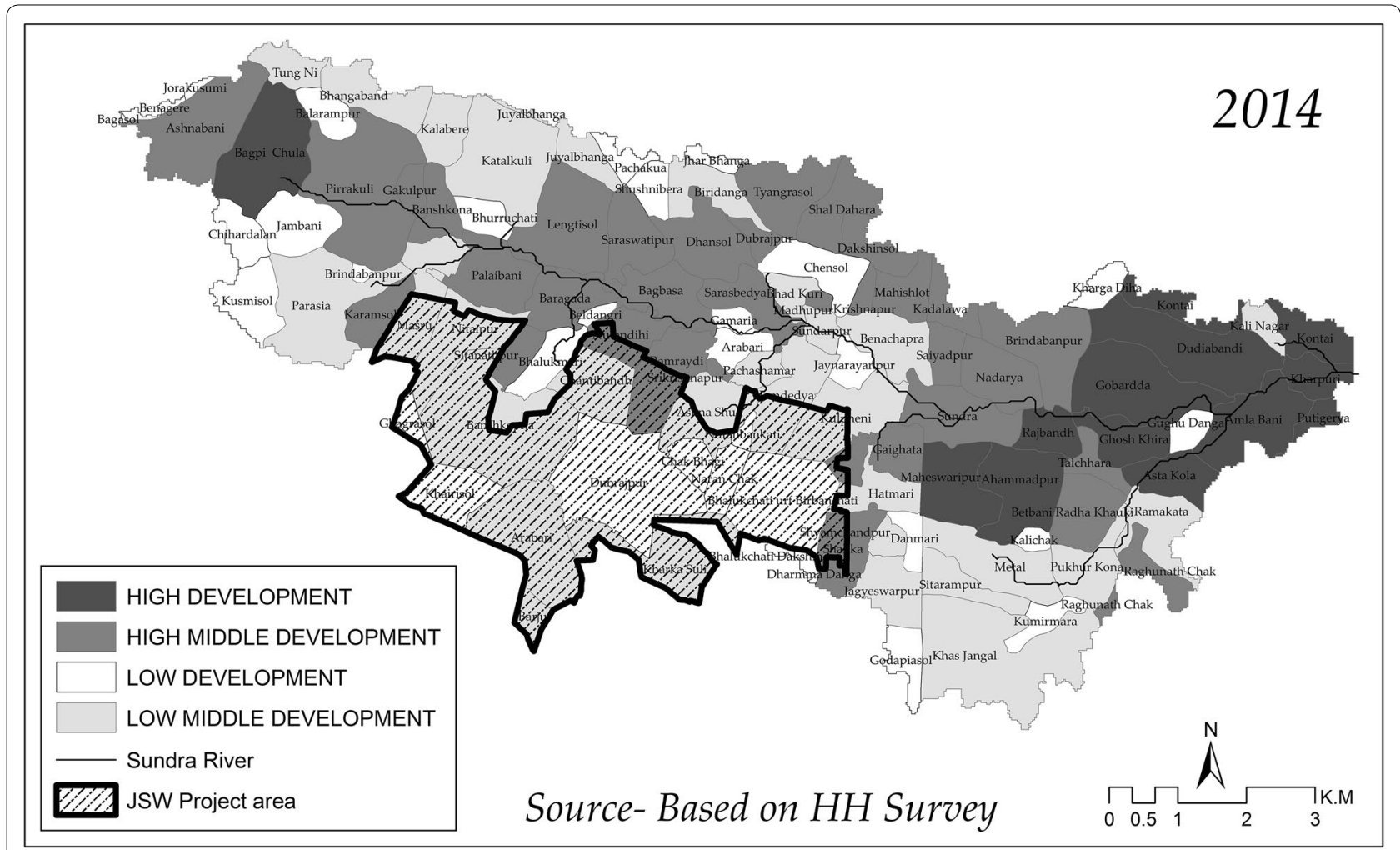

Fig. 8 Level of development in agricultural sector after land acquisition (2014)

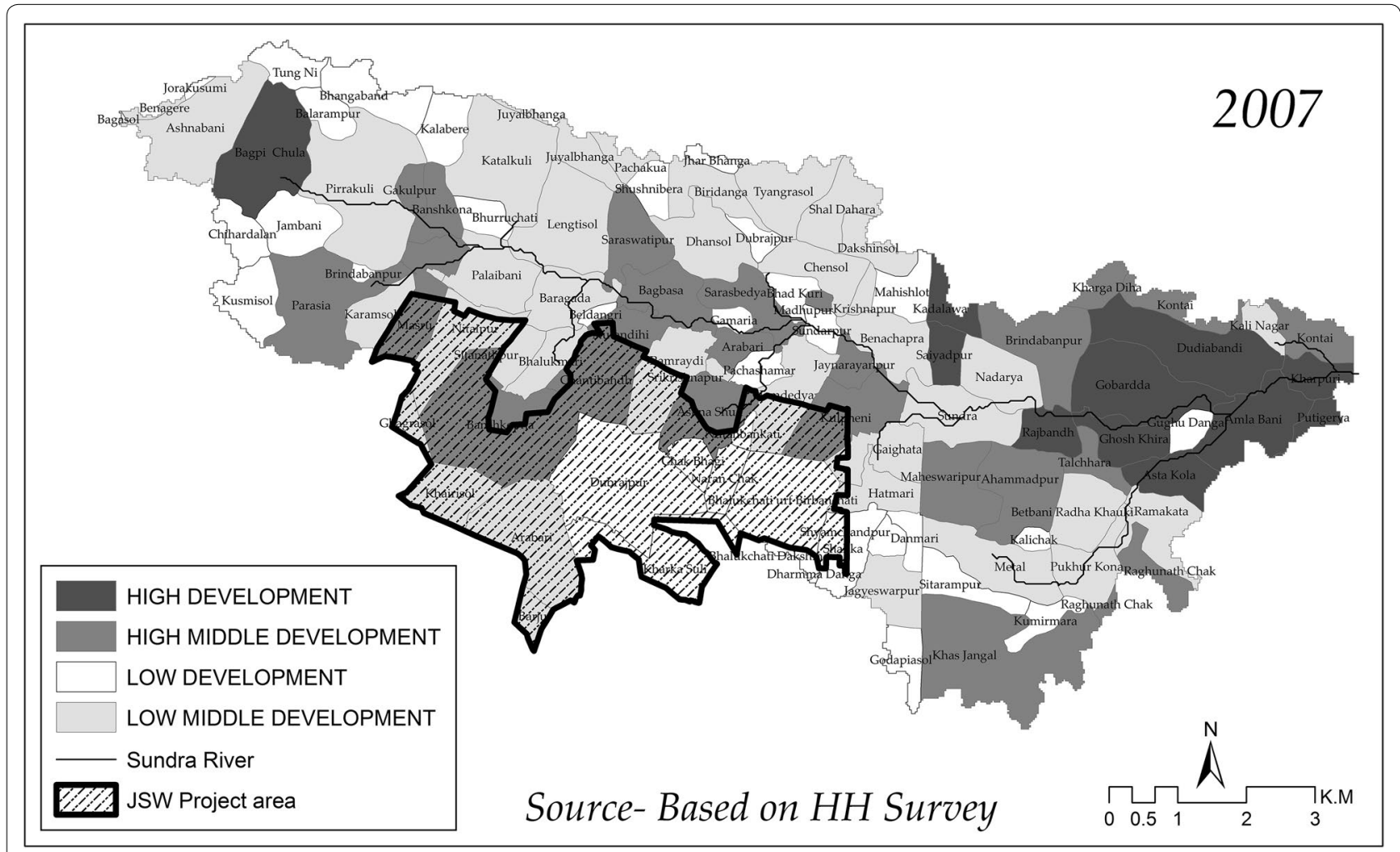

Fig. 9 Level of development in agricultural sector before land acquisition (2007) 
Table 4 Number of project affected mouzas with percentage of area and population under different level of agricultural development in 2014 and 2007. Source Author's own calculation

\begin{tabular}{|c|c|c|c|c|}
\hline Stages of development & Number of mouzas & Name of mouzas & $\%$ of total population & $\%$ of total area \\
\hline \multicolumn{5}{|l|}{ After land acquisition (2014) } \\
\hline High & $\mathrm{NIL}$ & $\mathrm{NIL}$ & NIL & $\mathrm{NIL}$ \\
\hline High middle & 4 & Nutandihi, Ramraydi, Gaighata, Shalika & 22.04 & 19.24 \\
\hline Low middle & 10 & $\begin{array}{l}\text { Masru, Nitaipur, Banshkopna, Chantibandh, Ashna Shuli, } \\
\text { Jamdedya, Kulpheni, Hatmari, Kharka Suli, Barju }\end{array}$ & 70.64 & 73.12 \\
\hline Low & 3 & Arabari, Khairisol, Ghagrasol & 7.32 & 7.64 \\
\hline \multicolumn{5}{|c|}{ Before land acquisition (2007) } \\
\hline High & 1 & Kharka Suli & 0.26 & 7.38 \\
\hline High middle & 6 & $\begin{array}{l}\text { Masru, Nutandihi, Banshkopna, Chantibandh, Ashna Shuli, } \\
\text { Kulpheni }\end{array}$ & 39.45 & 46.45 \\
\hline Low middle & 10 & $\begin{array}{l}\text { Nitaipur, Ramraydi, Jamdedya, Gaighata, Hatmari, Shalika, } \\
\text { Arabari, Khairisol, Barju, Ghagrasol }\end{array}$ & 60.30 & 46.17 \\
\hline Low & NIL & $\mathrm{NIL}$ & NIL & NIL \\
\hline
\end{tabular}

development category, $7.3 \%$ of total project affected population has been distributed in three mouzas namely Arabari, Khairisol and Ghagrasol and that covers by $7.6 \%$ of total project affected area in 2014. But in 2007 before acquisition no mouza was in this category. So, agricultural development has been observed outside of project affected mouzas. The major causes of low development in project site are reducing of farm size due to land acquisition and inadequate irrigation facilities that affected the paddy production adversely. Other causes of backwardness include poor quality of land, low area under commercial crop, low crop density, poor doses of fertilizer, etc. Action is required to be taken in these mouzas for improving the level of development in agriculture sector.

\section{Conclusion}

Economic planning at the grass root level is necessary for bringing about uniform regional development. The level of development of project affected and non-project affected mouzas is studied with the help of CI based on optimum combination of different development indicators. In order to get a clear picture of regional disparities, the level of development is assessed separately for agricultural sector, infrastructural sector and socio-economic sector using CI of level of development method by Narain et al. (2011). Extensive deterioration in the level of development is observed in the project areas after land acquisition. The maximum reduction in net sown area has been observed due to land acquisition within project area like as Dubrajpur (73.6\%), Chantibandh (87.1\%), Chak Bhagi (56.7\%), Ramraydi (51.4\%), Ashna Shuli (39.5\%) and Nitaipur (38.1\%) mouza. As a consequence farm size becomes small that leads to maximum share of marginal workers at Khairisol (90\%), Nutandihi (84.4\%), Arabari (75.3\%), Banshkopna (67.7\%), Chantibandh (58.1\%) and Ashna Shuli (61.2\%). Major reduction in crop income has been observed at Banshkopna (78.9\%), Ashna Shuli (79.9\%), Kharka Suli (77.4\%), Gaighata $(78.0 \%)$ at project site. At project affected mouzas more than $30 \%$ of agricultural workers are now being engaged in non-farm activities and try to reduce the livelihood vulnerability. Composite Index of agricultural development varies from 0.28 to 0.94 that indicates a greatest regional disparity exiting in agricultural development. After detailed analysis it has been observed that after acquisition (2014) $56.31 \%$ of total project area is in low and low middle development category while it was $43.06 \%$ before acquisition (2007). It implies that after closure of proposed project work majority of population now exists in low middle and low development category that means socioeconomic development has been stopped here. So there is an immediate need for proper planning on project affected mouzas to make alternative livelihood opportunities with help of local NGOs and Government.

\section{Abbreviations}

Mtpa: million ton per annum; Cl: composite index; NGO: Non-Government Organization; CPP: Captive Power Plant; MW: mega watt; km: kilo meter; sq: square; kg: kilo gram; SD: standard deviation.

\section{Authors' contributions}

SPS conceived of the study, carried out field work, analyzed data, drafted the manuscript and coordinate the whole work. RKM gave technical support and supervised the whole work, helped interpret the result and edited the paper. Both authors read and approved the final manuscript.

\section{Acknowledgements}

I wish to record my sincere gratitude to all the officials of all Departments including B.L.R.O (Salboni), D.L.R.O (Medinipur), Survey of India Government 
of West Bengal who had provided me valuable information on my study. I am highly obliged to Mr. Arun Mahato, local inhabitant of Salboni, Paschim Medinipur for assisting in field work and data analysis.

\section{Competing interests}

The authors declare that they have no competing interests.

\section{Availability of data and materials}

All relevant raw data will be freely available for non-commercial purposes.

\section{Consent of publication}

Not applicable.

Ethics approval and consent to participate

Not applicable.

\section{Funding}

Funding information is not applicable/No funding was received.

\section{Publisher's Note}

Springer Nature remains neutral with regard to jurisdictional claims in published maps and institutional affiliations.

Received: 10 April 2017 Accepted: 18 August 2017

Published online: 23 August 2017

\section{References}

Akama JS, Kieti D (2007) Tourism and socio-economic development in developing countries: a case study of Mombasa Resort in Kenya. J Sustain Tour 15(6):735-748

Anandabazar P (2014) Official announcement by JSW chairperson come managing director put the proposed JSW Bengal steel project hold. http:// www.anandabazar.com/searchresult/site-search-7.1881001?q=Salboni\& page $=3 \&$ short $=$ desc\&slab=0\&tnp $=3$. Accessed 4 Dec 2014

Arief S (1982) Regional disparities in Malaysia. Soc Indic Res 11(3):259-267

Bhatia VK, Rai SC (2004). Evaluation of socio-economic development in small areas. New Delhi, India. http://planningcommission.nic.in/reports/sereport/ser/std_smlarea.pdf. Accessed 6 Nov 2014

Bhattacharya N, Mahalanobis B (1967) Regional disparities in household consumption in India. J Am Stat Assoc 62 (317):143-161

Census of India (2001) India general population tables. Controller of Publication, Delhi

Census of India (2011) India general population tables. Controller of Publication, Delhi

Choudhury UDR (1992) Inter-state and intra-state variations in economic development and standard of living. Economic and Political Weekly, pp 2651-2655

Choudhury UDR (1995) National Income Accounting, Inter-Regional and IntraRegional Variations

Das A (1999) Socio-economic development in India: a regional analysis. Dev Soc 28(2):313-345

Dasgupta B (1971) Socio-economic classification of districts: a statistical approach. Economic and Political Weekly pp 1763-1774

Demurger S (2001) Infrastructure development and economic growth: an explanation for regional disparities in China? J Comp Econ 29(1):95-117

District statistical handbook (2006) Bureau of applied economics and statistics, Paschim Medinipur. Govt. of W.B, pp 1-91

District statistical handbook (2007) Bureau of applied Economics and statistics, Paschim Medinipur. Govt. of W.B, pp 1-91

Drewnowski J (1972) Social indicators and welfare measurement: remarks on methodology. J Dev Stud 8(3):77-90

Dreze J, Sen A (1999) India: economic development and social opportunity. OUP Catalogue

Ewusi K (1976) Disparities in levels of regional development in Ghana. Soc Indic Res 3(1):75-100

Florek K, Łukaszewicz J, Perkal J, Steinhaus H, Zubrzycki S (1951) Taksonomia wrocławska,'Przegląd Antropologiczny', vol XVII
Harbison FH, Maruhnic I, Rerwick JR (1968) An evaluation of human resources development indicators, Princeton

Hellwig Z (1967) Procedure of evaluating high-level man power data and typology of countries by means of the taxonomic method (Unpublished UNESCO working paper). http://unesdoc.unesco.org/ images/0001/000123/012349eo.pdf. Accessed 7 July 2014

Inglehart R (1997) Modernization and postmodernization: cultural, economic, and political change in 43 societies, vol 19. Princeton University Press, Princeton

Jindal Steel and Power Ltd (2014) ElA report on Greenfield Integrated Steel Plant (6MTPA) at Asnabani, Block: Potka, Jamshedpur District: East Singhbhum (Jharkhand). http://environmentclearance.nic.in/Search.aspx. Accessed 19 Nov 2014

JSW EIA (2007) Rapid environmental impact assessment for the proposed 3.0 Mtpa integrated steel plant at Godapiasal, Paschim Medinipur district, West Bengal. Vitma Labs Ltd, 142, IDA, Hyderbad

Long H, Heilig GK, Li X, Zhang M (2007) Socio-economic development and land-use change: analysis of rural housing land transition in the Transect of the Yangtse River, China. Land Use Policy 24(1):141-153

Madon S (2000) The Internet and socio-economic development: exploring the interaction. Inf Technol People 13(2):85-101

Myrdal G (1972) Asian drama; an inquiry into the poverty of nations, vol 2. http://www.jstor.org/stable/20634250

Narain P, Sharma SD, Rai SC, Bhatia VK (1991) Dimensions of socio-economic development in Jammu \& Kashmir. Development 1981:82

Narain P, Rai SC, Sarup S (1992) Classification of districts based on socio-economic development in Orissa. Yojana 36(23):9-12

Narain P, Rai SC, Sarup S (1993) Evaluation of economic development in Orissa. J Indian Soc Agric Stat 45(2):249-278

Narain P, Rai SC, Sarup S (1994a) Inter-districts disparities in socio-economic development in Kerala. J Indian Soc Agric Stat 46:362-377

Narain P, Rai SC, Sarup S (1994b) Regional dimensions of socio-economic development in Andhra Pradesh. J Indian Soc Agric Stat 46:156-165

Narain P, Rai SC, Sarup S (1995) Regional disparities in the levels of development in Uttar Pradesh. J Indian Soc Agric Stat 47(3):288-304

Narain P, Rai SC, Sarup S (1996) Dynamics of socio-economic development in Maharashtra. J Indian Soc Agric Stat 48:360-372

Narain P, Rai SC, Bhatia VK (1997) Regional pattern of socio-economic development in Karnataka. J Indian Soc Agric Stat 50:380-391

Narain P, Sharma SD, Rai SC, Bhatia VK (2000) Regional disparities in socioeconomic development in Tamil Nadu. J Indian Soc Agric Stat 53:35-46

Narain P, Sharma SD, Rai SC, Bhatia VK (2002) Dimensions of regional disparities in socio-economic development in Madhya Pradesh. J. Indian Soc Agric Stat 55:88-107

Narain P, Sharma SD, Rai SC, Bhatia VK (2003) Evaluation of economic development at micro level in Karnataka. J Indian Soc Agric Stat 56:52-63

Narain P, Sharma SD, Rai SC, Bhatia VK (2004) Estimation of socio-economic development in hilly States. J Ind Soc Agril Stat 58:126-135

Narain P, Sharma SD, Rai SC, Bhatia VK (2005) Estimation of socio-economic development of different districts in Kerala. J Indian Soc Agric Stat 59:48-55

Narain P, Sharma SD, Rai SC, Bhatia VK (2009) Inter-district variation of socio-economic development in Andhra Pradesh. J Indian Soc Agric Stat 63(1):35-42

Narain P, Bhatia VK, Rai SC (2011) Pattern of regional disparities in Socio-economic development in West Bengal. J Indian Soc Agric Stat 65(1):27-35

Ohlan R (2013) Pattern of regional disparities in socio-economic development in India: district level analysis. Soc Indic Res 114(3):841-873

Parihar HPS, Srivastava S (2003) Statistical measures of development for an ideal village. Ind J Ext Educ 3:100-103

Rai SC, Bhatia VK (2004) Dimensions of regional disparities in socio-economic development of Assam. J Indian Soc Agric Stat 57:178-190

Rai SC, Bhatia VK (2011) Pattern of regional disparities in socio-economic development in West Bengal. J Indian Soc Agric Stat 65(1):27-35

Raja TA, Yousaf T (2014) Statistical evaluation of socio-economic development with a new composite index of Kashmir valley. Int Res J Agric Econ Stat 5(2):317-320

Sarker PC (1994) Regional imbalances in Indian economy over plan periods. Economic and Political Weekly pp 621-633

Sudalaimuthu S, RAJ SA (2009) Logistics management for international business: text and cases. PHI Learning Pvt, Ltd

Szirmai A (2015) Socio-economic development. Cambridge University Press, Cambridge 